\title{
Deviation of inverse square law based on Dunkl derivative: deformed Coulomb's law
}

\author{
Y. $\operatorname{Kim}^{a}$, W. Sang Chung ${ }^{a, \dagger}$ and H. Hassanabadi ${ }^{b, \ddagger}$ \\ ${ }^{a}$ Department of Physics and Research Institute of Natural Science, \\ College of Natural Science, Gyeongsang National University, Jinju 660-701, Korea. \\ ${ }^{b}$ Faculty of physics, Shahrood University of Technology, Shahrood, Iran \\ e-mail: ${ }^{\dagger}$ mimip44@naver.com; ${ }^{\ddagger}$ h.hasanabadi@shahroodut.ac.ir
}

Received 18 March 2020; accepted 19 March 2020

In this paper, we consider the Coulomb's law with deviation. We use the Dunkl derivative to derive the deformed Gauss law for the electric field and the electrostatic potential, which gives a new deformed electrostatics called a Dunkl-deformed electrostatics. We modify the Dunkl derivative for the electric field for multi-sources or continuous charge distribution. We discuss some examples of the Dunkl-deformed electrostatics.

Keywords: Coulomb's law; Dunkl derivative; deformed Gauss law.

PACS: 03.65.Ge; 03.65.Fd; 02.30.Gp.

DOI: https://doi.org/10.31349/RevMexFis.66.411

\section{Introduction}

The inverse square law arises in the Coulomb's law. This states that the electric field at $\mathbf{r}$ due to the charge $q$ located at origin is given by

$$
\mathbf{E}(\mathbf{r})=\frac{q}{|\mathbf{r}|^{2}} \hat{\mathbf{r}}=\frac{q}{|\mathbf{r}|^{3}} \mathbf{r} .
$$

Recently, some measurements were accomplished for the accuracy of inverse square law for Coulomb's law by considering the small deviation of the inverse square law of the form

$$
\mathbf{E}(\mathbf{r})=\frac{q}{|\mathbf{r}|^{2+\sigma}} \hat{\mathbf{r}}=\frac{q}{|\mathbf{r}|^{3+\sigma}} \mathbf{r},
$$

where $\sigma$ can be regarded as a deviation from the inverse square law [1-3]. Plimpton and Lawton [1] charged an outer sphere with a slowly varying alternating current and detected the potential difference between the inner and outer spheres. They found $\sigma=2 \times 10^{-9}$. In 1970, Bartlett, Goldhagen, and Phillips [2] also achieved an upper limit of $\sigma=1.3 \times 10^{-13}$. One year later, Williams and Faller [3] estimated $\sigma=(2.7 \pm 3.1) \times 10^{-16}$. From these experimental data for $\sigma$, we know that the deviation from the inverse square law is sufficiently small but can be considered to be non-zero.

As a theoretical background of the Eq. (2), we will invoke Wigner deformation. In 1950, Wigner [4] proposed a new deformed Heisenberg algebra of the form

$$
[\hat{x}, \hat{p}]=i(1+2 \nu R), \quad \nu \in \mathbb{R},
$$

where $R$ is the reflection operator obeying

$$
R \hat{x}=-\hat{x} R,
$$

and we set $\hbar=1$. It is well known that algebra (3) gives the same equation of motion for the harmonic potential, which implies that algebra is the general second quantization scheme for the classical harmonic potential problem. The coordinate representation for the Eq. (3) is then given by

$$
\hat{p}=\frac{1}{i} D_{x}, \quad \hat{x}=x,
$$

where Dunkl derivative (DD) [5] $D_{x}$ is given by

$$
D_{x}=\partial_{x}+\frac{\nu}{x}\left(1-R_{x}\right)
$$

and the reflection operator obeys

$$
R_{x} f(x)=f(-x) .
$$

It is well known that Wigner algebra (3) is linked to the two-particle Calogero model [6-8] when the Wigner parameter is related to the Calogero coupling constant. Wigner Hamiltonian, which comes from Wigner algebra, has the potential which consists of a simple harmonic potential and an additional inverse square potential. This potential was shown to possess the conformal symmetry and has been the subject of much interest [9-14]. Especially, Macfarlane [14] found the relation between Wigner algebra and para-statistics.

Here we have a fundamental question. If Coulomb's law does not obey the inverse square law, what will happen? Is it possible to construct the electrostatics in such case? The answer is YES, which is the main purpose of this work. In this paper, we use DD to derive the deviation of the inverse square law. We apply the deviation of the inverse square law to the electrostatics. This paper is organized as follows. In Sec. 2, we discuss the deformed electrostatics with DD. In Sec. 3, we discuss the modification of DD and deformed electrostatics when the point charge is not located at origin. In Sec. 4, we discuss the deformed electrostatics with a multi-source and continuous source. In Sec. 5, we discuss some examples of electrostatics with discrete charges. In Sec. 6, we discuss some examples of Dunkl-deformed electrostatics with continuous charge distribution. 


\section{Deformed electrostatics with DD}

In this section, we consider the electrostatics with DD. To do so, we introduce the vector DD's as

$$
\mathbf{D}=\nabla+\nu \mathbf{P}
$$

where

$$
\begin{aligned}
\mathbf{P} & =\hat{\mathbf{e}}_{1} \frac{1}{x_{1}}\left(1-R_{1}\right) \\
& +\hat{\mathbf{e}}_{\mathbf{2}} \frac{1}{x_{2}}\left(1-R_{2}\right)+\hat{\mathbf{e}}_{\mathbf{3}} \frac{1}{x_{3}}\left(1-R_{3}\right)
\end{aligned}
$$

and

$$
R_{i} x_{j}=\left\{\begin{array}{ll}
-x_{j} R_{i} & (i=j) \\
x_{j} R_{i} & (i \neq j)
\end{array} .\right.
$$

We consider the electromagnetic theory in which the vector differential operator $\nabla$ is replaced with the vector DD operator D. Outside the point charge $q$ located at the origin, the electric field is assumed to obey the deformed Gauss law outside source

$$
\mathbf{D} \cdot \mathbf{E}=0 \text {. }
$$

Now let us solve the Eq. (11) by setting

$$
\mathbf{E}=f(r) \mathbf{r},
$$

where

$$
r=|\mathbf{r}|=\sqrt{x_{1}^{2}+x_{2}^{2}+x_{3}^{2}} .
$$

Inserting the Eq. (12) into the Eq. (11) we get

$$
\mathbf{D} \cdot \mathbf{E}=r f^{\prime}(r)+(3+6 \nu) f(r)=0 .
$$

Solving the Eq. (14) we get

$$
f(r)=\frac{q}{r^{3+6 \nu}} .
$$

Thus, the deformed electric field reads

$$
\mathbf{E}=\frac{q}{r^{3+6 \nu}} \mathbf{r}
$$

which reveals the law of inverse square with deviation. One can easily check that the deformed electric field obeys

$$
\mathbf{D} \times \mathbf{E}=0,
$$

which holds because $\mathbf{P} \times \mathbf{E}=0$. Thus, the electrostatic potential is defined through

$$
\mathbf{E}=-\mathbf{D} V(r)=-\nabla V(r) .
$$

Now let us derive the deformed Gauss law. We assume that the Gauss law for a point charge located at the origin takes the form

$$
\int(\mathbf{D} \cdot \mathbf{E}) \mu(r) d^{3} \mathbf{r}=4 \pi q
$$

where $\mu(r)$ is the weight function. The Eq. (19) can be written as

$$
\int(\nabla \cdot \mathbf{E}) \mu(r) d^{3} \mathbf{r}+\nu \int(\mathbf{P} \cdot \mathbf{E}) \mu(r) d^{3} \mathbf{r}=4 \pi q
$$

or

$$
\begin{gathered}
\int \nabla \cdot(\mathbf{E} \mu(r)) d^{3} \mathbf{r}-\int \mathbf{E} \cdot \mu^{\prime}(r) \frac{\mathbf{r}}{r} d^{3} \mathbf{r} \\
+\nu \int(\mathbf{P} \cdot \mathbf{E}) \mu(r) d^{3} \mathbf{r}=4 \pi q
\end{gathered}
$$

Inserting the Eq. (16) into the Eq. (21) we get

$$
4 \pi q r^{-6 \nu} \mu(r)+q \int\left(\frac{6 \nu \mu(r)}{r^{3}}-\frac{\mu^{\prime}(r)}{r^{2}}\right) d^{3} \mathbf{r}=4 \pi q,
$$

which gives

$$
\mu(r)=r^{6 \nu}
$$

Thus, the deformed Gauss law reads

$$
r^{6 \nu} \mathbf{D} \cdot \mathbf{E}=4 \pi \rho(\mathbf{r})
$$

where $\rho$ denotes the charge density, and for a point charge it is

$$
\rho(\mathbf{r})=q \delta(\mathbf{r})
$$

If we locate a charge $Q$ on $\mathbf{r}$, it will be subject to the force of the form

$$
\mathbf{F}=Q \mathbf{E}=\frac{q Q}{|\mathbf{r}|^{3+6 \nu}} \mathbf{r}
$$

Now let us find the potential energy for the deformed Coulomb force (26). The Eq. (26) indicates that the deformed Coulomb force is also conservative. Thus, the potential energy is defined through

$$
\mathbf{F}=-\nabla U(r)
$$

Solving the Eq. (27) we get

$$
U(r)=\frac{q Q}{(1+6 \nu)|\mathbf{r}|^{1+6 \nu}} .
$$

Similarly, the electrostatic potential $V$ is defined through

$$
\mathbf{E}=-\nabla V(r)
$$

which gives

$$
V(r)=\frac{q}{(1+6 \nu)|\mathbf{r}|^{1+6 \nu}}
$$

Thus, the electrostatic potential obeys the Dunkl-Laplace equation

$$
\mathbf{D} \cdot \nabla V=0 \text {. }
$$




\section{Modification of DD and deformed electro- statics when the point charge is not located at the origin}

In ordinary electro-magnetics, consider the Coulomb force. Let $\mathbf{F}$ denote the force acting on a electrically charged particle, with charge $q$ located at $\mathbf{r}$, due to the presence of a charge $q^{\prime}$ located at $\mathbf{r}^{\prime}$. According to Coulomb's law, this force is, in a vacuum, given by the expression

$$
\begin{aligned}
\mathbf{F} & =\frac{q q^{\prime}\left(\mathbf{r}-\mathbf{r}^{\prime}\right)}{\left|\mathbf{r}-\mathbf{r}^{\prime}\right|^{3}}=-q q^{\prime} \nabla\left(\frac{1}{\left|\mathbf{r}-\mathbf{r}^{\prime}\right|}\right) \\
& =q q^{\prime} \nabla^{\prime}\left(\frac{1}{\left|\mathbf{r}-\mathbf{r}^{\prime}\right|}\right) .
\end{aligned}
$$

The vector DD defined in the Eq. (8) does not obey the above property. Thus, we need a new definition for vector DD when we consider two points, source point, and field point, and this derivative is acted on the function in $\mathbf{r}-\mathbf{r}^{\prime}$. Here we define the DD gradient of the function $f\left(\left|\mathbf{r}-\mathbf{r}^{\prime}\right|\right)$ as

$$
\operatorname{DDgrad} f\left(\left|\mathbf{r}-\mathbf{r}^{\prime}\right|\right)=\mathbf{D}_{\mathbf{r}, \mathbf{r}^{\prime}} f\left(\left|\mathbf{r}-\mathbf{r}^{\prime}\right|\right),
$$

where

$$
\mathbf{D}_{\mathbf{r}, \mathbf{r}^{\prime}}=\nabla+\nu \mathbf{P}_{\mathbf{r}, \mathbf{r}^{\prime}}
$$

and

$$
\left(\mathbf{P}_{\mathbf{r}, \mathbf{r}^{\prime}}\right)_{i}=\frac{1}{x_{i}-x_{i}^{\prime}}\left(1-R_{x_{i}, x_{i}^{\prime}}\right),
$$

and the exchange operator obeys

$$
R_{x_{i} x_{i}^{\prime}}\left(x_{i}-x_{i}^{\prime}\right)=\left(x_{i}^{\prime}-x_{i}\right) R_{x_{i} x_{i}^{\prime}} .
$$

Similarly, we define the DD divergence, and DD curl acted on the vector function of the form $f\left(\left|\mathbf{r}-\mathbf{r}^{\prime}\right|\right)\left(\mathbf{r}-\mathbf{r}^{\prime}\right)$ as

$$
\begin{gathered}
\operatorname{DDdiv}\left(f\left(\left|\mathbf{r}-\mathbf{r}^{\prime}\right|\right)\left(\mathbf{r}-\mathbf{r}^{\prime}\right)\right)=\mathbf{D}_{\mathbf{r}, \mathbf{r}^{\prime}} \\
\cdot\left(f\left(\left|\mathbf{r}-\mathbf{r}^{\prime}\right|\right)\left(\mathbf{r}-\mathbf{r}^{\prime}\right)\right)
\end{gathered}
$$

and

$$
\begin{aligned}
\operatorname{DDcurl} & \left(f\left(\left|\mathbf{r}-\mathbf{r}^{\prime}\right|\right)\left(\mathbf{r}-\mathbf{r}^{\prime}\right)\right)=\mathbf{D}_{\mathbf{r}, \mathbf{r}^{\prime}} \\
& \times\left(f\left(\left|\mathbf{r}-\mathbf{r}^{\prime}\right|\right)\left(\mathbf{r}-\mathbf{r}^{\prime}\right)\right) .
\end{aligned}
$$

The electric field at $\mathbf{r}$ due to charge $q^{\prime}$ located at $\mathbf{r}^{\prime}$ is then given by

$$
\mathbf{E}\left(\mathbf{r}-\mathbf{r}^{\prime}\right)=\frac{q^{\prime}}{\left|\mathbf{r}-\mathbf{r}^{\prime}\right|^{3+6 \nu}}\left(\mathbf{r}-\mathbf{r}^{\prime}\right),
$$

which satisfies

$$
\begin{aligned}
\left|\mathbf{r}-\mathbf{r}^{\prime}\right|{ }^{6 \nu} \mathbf{D}_{\mathbf{r}, \mathbf{r}^{\prime}} \cdot \mathbf{E}\left(\mathbf{r}-\mathbf{r}^{\prime}\right) & =4 \pi q^{\prime} \delta\left(\mathbf{r}-\mathbf{r}^{\prime}\right) \\
\mathbf{D}_{\mathbf{r}, \mathbf{r}^{\prime}} \times \mathbf{E}\left(\mathbf{r}-\mathbf{r}^{\prime}\right) & =0 .
\end{aligned}
$$

The deformed electrostatic potential is given by

$$
\mathbf{E}\left(\mathbf{r}-\mathbf{r}^{\prime}\right)=-\nabla V\left(\left|\mathbf{r}-\mathbf{r}^{\prime}\right|\right),
$$

which gives

$$
V\left(\left|\mathbf{r}-\mathbf{r}^{\prime}\right|\right)=\frac{q^{\prime}}{1+6 \nu}\left(\frac{1}{\left|\mathbf{r}-\mathbf{r}^{\prime}\right|^{1+6 \nu}}\right) .
$$

\section{Deformed electrostatics with multi source and continuous source}

Now let us consider the multi source case. Consider the situation that there are $N$ charges $q_{1}, q_{2}, \cdots, q_{N}$, each of which is located at $\mathbf{r}_{1}, \mathbf{r}_{2}, \cdots, \mathbf{r}_{N}$. The electric field at $\mathbf{r}$ due to $N$ charges is defined as the sum of $N$ sub electric fields as follows:

$$
\mathbf{E}(\mathbf{r})=\sum_{j=1}^{N} \mathbf{E}\left(\mathbf{r}-\mathbf{r}_{j}\right),
$$

where the sub electric field due to charge $q_{j}$ is

$$
\mathbf{E}\left(\mathbf{r}-\mathbf{r}_{j}\right)=\frac{q_{j}}{\left|\mathbf{r}-\mathbf{r}_{j}\right|^{3+6 \nu}}\left(\mathbf{r}-\mathbf{r}_{j}\right) .
$$

The sub electric field obeys

$$
\left|\mathbf{r}-\mathbf{r}_{j}\right|^{6 \nu} \mathbf{D}_{\mathbf{r}, \mathbf{r}_{j}} \cdot \mathbf{E}\left(\mathbf{r}-\mathbf{r}_{j}\right)=4 \pi q_{j} \delta\left(\mathbf{r}-\mathbf{r}_{j}\right),
$$

and

$$
\mathbf{D}_{\mathbf{r}, \mathbf{r}_{j}} \times \mathbf{E}\left(\mathbf{r}-\mathbf{r}_{j}\right)=0 .
$$

The electrostatic potential is defined through

$$
\mathbf{E}_{t o t}(\mathbf{r})=-\nabla V(r),
$$

which gives

$$
V(r)=\sum_{j=1}^{N} \frac{q_{j}}{(1+6 \nu)\left|\mathbf{r}-\mathbf{r}_{j}\right|^{1+6 \nu}} .
$$

If the discrete electric charges are small and numerous enough, we introduce the electric charge density $\rho$ located at $\mathbf{r}^{\prime}$ within a volume $V^{\prime}$ of a limited extent and replace summation with integration over this volume. This allows us to describe the total electric field as integral of the infinitesimal electric field as follows:

$$
\mathbf{E}(\mathbf{r})=\int_{V^{\prime}} d^{3} \mathbf{r}^{\prime} \mathbf{E}\left(\mathbf{r}-\mathbf{r}^{\prime}\right),
$$

where the infinitesimal electric field due to the infinitesimal charge located at $\mathbf{r}^{\prime}$ is

$$
\mathbf{E}\left(\mathbf{r}-\mathbf{r}^{\prime}\right)=\rho\left(\mathbf{r}^{\prime}\right) \frac{\mathbf{r}-\mathbf{r}^{\prime}}{\left|\mathbf{r}-\mathbf{r}^{\prime}\right|^{3+6 \nu}} .
$$

The infinitesimal electric filed obeys

$$
\left|\mathbf{r}-\mathbf{r}^{\prime}\right|^{6 \nu} \mathbf{D}_{\mathbf{r}, \mathbf{r}^{\prime}} \cdot \mathbf{E}\left(\mathbf{r}-\mathbf{r}^{\prime}\right)=4 \pi \rho\left(\mathbf{r}^{\prime}\right) \delta\left(\mathbf{r}-\mathbf{r}^{\prime}\right),
$$

and

$$
\mathbf{D}_{\mathbf{r}, \mathbf{r}^{\prime}} \times \mathbf{E}\left(\mathbf{r}-\mathbf{r}^{\prime}\right)=0 .
$$

The electrostatic potential is defined through

$$
\mathbf{E}(\mathbf{r})=-\nabla V(r),
$$

which gives

$$
V(r)=\int_{V^{\prime}} \frac{\rho\left(\mathbf{r}^{\prime}\right)}{1+6 \nu}\left(\frac{1}{\left|\mathbf{r}-\mathbf{r}^{\prime}\right|^{1+6 \nu}}\right) .
$$




\section{Some examples of Dunkl-deformed electro- statics with discrete charges}

Now let us discuss some examples of Dunkl-deformed electrostatics with discrete charges.

\subsection{Monopole}

Let us consider the case that a charge $q$ is located at $a \mathbf{k}$, where $\mathbf{k}$ denotes the unit vector in $z$ direction. Then the electrostatic potential is

$$
V=\frac{q}{1+6 \nu}\left(\frac{1}{|\mathbf{r}-a \mathbf{k}|^{1+6 \nu}}\right)
$$

and the electric field is

$$
\mathbf{E}=-\nabla V=\frac{q(\mathbf{r}-a \mathbf{k})}{|\mathbf{r}-a \mathbf{k}|^{3+6 \nu}}
$$

If we express the Eq. (56) in terms of the spherical coordinates, we get

$$
V=\frac{q}{\left(r^{2}+a^{2}-2 a r \cos \theta\right)^{1 / 2+3 \nu}} .
$$

Let us consider the Gegenbauer polynomial defined through the following generating function

$$
\frac{1}{\left(1-2 x t+t^{2}\right)^{\alpha}}=\sum_{n=0}^{\infty} C_{n}^{(\alpha)}(x) t^{n} .
$$

The generating function for Gegenbauer polynomial is a generalization of the generating function for Legendre polynomials. Indeed, when $\alpha=1 / 2$, the Gegenbauer polynomials become Legendre polynomials. Thus, with the help of generating function for Gegenbauer polynomials, the electrostatic potential reads

$$
V=\frac{q}{(1+6 \nu) r} \sum_{n=0}^{\infty} C_{n}^{(1 / 2+3 \nu)}(\cos \theta)\left(\frac{a}{r}\right)^{n}
$$

for $r>a$, while we get

$$
V=\frac{q}{(1+6 \nu) a} \sum_{n=0}^{\infty} C_{n}^{(1 / 2+3 \nu)}(\cos \theta)\left(\frac{r}{a}\right)^{n}
$$

for $r<a$. We know that the Gegenbauer polynomial satisfies the recurrence relation

$$
\begin{aligned}
C_{0}^{\alpha}(x) & =1 \\
C_{1}^{\alpha}(x) & =2 \alpha x \\
C_{n}^{\alpha}(x) & =\frac{1}{n}\left[2 x(n+\alpha-1) C_{n-1}^{\alpha}(x)\right. \\
& \left.-(n+2 \alpha-2) C_{n-2}^{\alpha}(x)\right] .
\end{aligned}
$$

\subsection{Dipole}

Let us consider the case that a charge $q$ is located at $a \mathbf{k}$, and another charge $-q$ is located at $-a \mathbf{k}$. Then the electrostatic potential is composed of the electrostatic potential due to $q$, $V_{q}$, and the electrostatic potential due to $-q, V_{-q}$,

$$
\begin{aligned}
V & =\frac{q}{1+6 \nu}\left(\frac{1}{|\mathbf{r}-a \mathbf{k}|^{1+6 \nu}}\right) \\
& -\frac{q}{1+6 \nu}\left(\frac{1}{|\mathbf{r}+a \mathbf{k}|^{1+6 \nu}}\right) .
\end{aligned}
$$

Thus, the electric field is

$$
\mathbf{E}=-\nabla V=\frac{q(\mathbf{r}-a \mathbf{k})}{|\mathbf{r}-a \mathbf{k}|^{3+6 \nu}}-\frac{q(\mathbf{r}+a \mathbf{k})}{|\mathbf{r}+a \mathbf{k}|^{3+6 \nu}} .
$$

If we express the Eq. (65) in terms of the spherical coordinates we get

$$
\begin{aligned}
V & =\frac{q}{1+6 \nu}\left[\frac{1}{\left(r^{2}+a^{2}-2 a r \cos \theta\right)^{1 / 2+3 \nu}}\right. \\
& \left.-\frac{1}{\left(r^{2}+a^{2}+2 a r \cos \theta\right)^{1 / 2+3 \nu}}\right] .
\end{aligned}
$$

The electric field is then given by

$$
\mathbf{E}=E_{r} \hat{r}+E_{\theta} \hat{\theta}
$$

where

$$
\begin{gathered}
E_{r}=q\left[\frac{r-a \cos \theta}{\left(r^{2}+a^{2}-2 a r \cos \theta\right)^{3 / 2+3 \nu}}\right. \\
\left.-\frac{r+a \cos \theta}{\left(r^{2}+a^{2}+2 a r \cos \theta\right)^{3 / 2+3 \nu}}\right] \\
E_{\theta}=q a \sin \theta\left[\frac{1}{\left(r^{2}+a^{2}+2 a r \cos \theta\right)^{3 / 2+3 \nu}}\right. \\
\left.+\frac{1}{\left(r^{2}+a^{2}-2 a r \cos \theta\right)^{3 / 2+3 \nu}}\right] .
\end{gathered}
$$

For $r \gg a$, we have

$$
V \approx \frac{p \cos \theta}{r}
$$

where the electric dipole moment is $p=2 q a$. Thus we have the same form as ordinary electro-magnetics.

\subsection{Quadrupole}

Let us consider the case that a charge $q$ is located at $a \mathbf{k}$, another charge $q$ is located at $-a \mathbf{k}$, and the third charge $-2 q$ at origin. Then the electrostatic potential is

$$
\begin{aligned}
V & =-\frac{2 q}{1+6 \nu}\left(\frac{1}{|\mathbf{r}|^{1+6 \nu}}\right)+\frac{q}{1+6 \nu}\left(\frac{1}{|\mathbf{r}-a \mathbf{k}|^{1+6 \nu}}\right) \\
& +\frac{q}{1+6 \nu}\left(\frac{1}{|\mathbf{r}+a \mathbf{k}|^{1+6 \nu}}\right) .
\end{aligned}
$$


Thus, the electric field is

$$
\begin{aligned}
\mathbf{E} & =-\nabla V=\frac{q(\mathbf{r}-a \mathbf{k})}{|\mathbf{r}-a \mathbf{k}|^{3+6 \nu}} \\
& +\frac{q(\mathbf{r}+a \mathbf{k})}{|\mathbf{r}+a \mathbf{k}|^{3+6 \nu}}-\frac{2 q \mathbf{r}}{|\mathbf{r}|^{3+6 \nu}} .
\end{aligned}
$$

If we express the Eq. (72) in terms of the spherical coordinates we get

$$
\begin{aligned}
V & =\frac{q}{1+6 \nu}\left[\frac{1}{\left(r^{2}+a^{2}-2 a r \cos \theta\right)^{1 / 2+3 \nu}}\right. \\
& \left.+\frac{1}{\left(r^{2}+a^{2}+2 a r \cos \theta\right)^{1 / 2+3 \nu}}-\frac{2}{r^{1+6 \nu}}\right] .
\end{aligned}
$$

The electric field is then given by

$$
\mathbf{E}=E_{r} \hat{r}+E_{\theta} \hat{\theta}
$$

where

$$
\begin{aligned}
E_{r} & =q\left[\frac{2}{r^{2+6 \nu}}-\frac{r-a \cos \theta}{\left(r^{2}+a^{2}-2 a r \cos \theta\right)^{3 / 2+3 \nu}}\right. \\
& \left.-\frac{r+a \cos \theta}{\left(r^{2}+a^{2}+2 a r \cos \theta\right)^{3 / 2+3 \nu}}\right] \\
E_{\theta} & =q a \sin \theta\left[\frac{1}{\left(r^{2}+a^{2}-2 a r \cos \theta\right)^{3 / 2+3 \nu}}\right. \\
& \left.+\frac{1}{\left(r^{2}+a^{2}+2 a r \cos \theta\right)^{3 / 2+3 \nu}}\right] .
\end{aligned}
$$

For $r \gg a$, we have

$$
V \approx \frac{Q}{r^{3}}\left[\left(\frac{3}{2}+3 \nu\right) \cos ^{2} \theta-\frac{1}{2}\right],
$$

where the electric quadrupole moment is $Q=2 q a^{2}$. Thus we know that the $\theta$-dependency of the potential is deformed.

\section{Some examples of Dunkl-deformed electro- statics with continuous charge distribution}

Let us discuss some examples of Dunkl-deformed electrostatics with continuous charge distribution.

\subsection{Electric field on the axis of a finite line charge}

A charge $Q$ is uniformly distributed along the $x$ axis from $x=0$ to $x=L>0$. The linear charge density for this charge is $\lambda=Q / L$. We wish to find the electric field produced by this line charge at some field point $P(x)$ with $x>L$ on the $x$-axis. The electrostatic potential is then given by

$$
\begin{aligned}
V_{P}(x) & =\frac{1}{1+6 \nu} \int_{y=0}^{L} \frac{\lambda d y}{|x-y|^{1+6 \nu}} \\
& =\frac{\lambda}{6 \nu(1+6 \nu)}\left[(x-L)^{-6 \nu}-x^{-6 \nu}\right] .
\end{aligned}
$$

For a small $\nu$, we get

$$
\begin{aligned}
V_{P}(x) & \approx \lambda \ln \left(\frac{x}{x-L}\right) \\
& +3 \lambda \nu \ln \left(\frac{x-L}{x}\right)[2+\ln (x(x-L))] .
\end{aligned}
$$

For $x \gg L$ we have

$$
V_{P}(x) \approx \frac{\lambda L}{(1+6 \nu) x^{1+6 \nu}} .
$$

The electric field is

$$
\begin{aligned}
E_{P}(x) & =-\frac{d}{d x} V(x) \\
& =\frac{\lambda}{1+6 \nu}\left[\frac{1}{(x-L)^{1+6 \nu}}-\frac{1}{x^{1+6 \nu}}\right] .
\end{aligned}
$$

For a small $\nu$, we get

$$
\begin{aligned}
E_{P}(x) & \approx \frac{\lambda L}{x(x-L)}-\frac{6 \nu}{x(x-L)} \\
& \times\left(L+L \ln x+x \ln \left(\frac{x-L}{x}\right)\right) .
\end{aligned}
$$

We can see that if $x$ is much larger than $L$, the electric field at $P$ is approximately

$$
E_{P} \approx \frac{Q}{x^{2+6 \nu}}
$$

That is, if we are sufficiently far away from the line charge, it approaches that of a point charge $Q$ at the origin.

\subsection{Electric field on the perpendicular bisector of a uni- formly charged line segment}

A charge $Q$ is uniformly distributed on a straight-line segment of length $L$ on the $y$-axis. The charge density is given by

$$
\lambda(y)=\left\{\begin{array}{ll}
\lambda & (|y| \leq L / 2) \\
0 & (|y|>L / 2)
\end{array} .\right.
$$

Now let us find the electrostatic potential and electric field at point $P$ on the $x$-axis, $P(x, 0)$, where we consider the case of $x>0$. The electrostatic potential is

$$
V_{P}=\frac{1}{1+6 \nu} \int_{y=-L / 2}^{L / 2} \frac{\lambda d y}{\left|\mathbf{r}-\mathbf{r}^{\prime}\right|^{1+6 \nu}},
$$

where $\mathbf{r}=x \mathbf{i}, \mathbf{r}^{\prime}=y \mathbf{j},(|y| \leq L / 2)$. Then we have

$$
\begin{aligned}
V_{P} & =\frac{2 \lambda}{1+6 \nu} \int_{y=0}^{L / 2} \frac{d y}{\left(x^{2}+y^{2}\right)^{\frac{1}{2}+3 \nu}} \\
& =\frac{\lambda L}{(1+6 \nu) x^{1+6 \nu}} 2 F_{1}\left(\frac{1}{2}+3 \nu, \frac{1}{2} ; \frac{3}{2} ; \frac{L^{2}}{4 x^{2}}\right) .
\end{aligned}
$$


For $x \gg L$ we have

$$
V_{P}(x) \approx \frac{\lambda L}{(1+6 \nu) x^{1+6 \nu}} .
$$

The electric field is

$$
\begin{aligned}
E_{P}(x) & =-\frac{d}{d x} V(x) \\
& =\frac{\lambda L}{x^{2+6 \nu}}{ }_{2} F_{1}\left(\frac{3}{2}+3 \nu, \frac{1}{2} ; \frac{3}{2} ; \frac{L^{2}}{4 x^{2}}\right) .
\end{aligned}
$$

We can see that if $x$ is much larger than $L$, the electric field at $P$ is approximately

$$
E_{P} \approx \frac{Q}{x^{2+6 \nu}} .
$$

\subsection{Electric field on the axis of a uniformly charged disk}

Consider a uniformly charged disk of radius $R$ centered at the origin and total charge $Q$, which lies on $x y$-plane. We can calculate the electrostatic potential and electric field at the point $P(0,0, z)$ on the $z$-axis with $z>0$. In this case, the surface charge density (the charge per unit area) is given by $\sigma=Q /\left(\pi R^{2}\right)$. Then the electrostatic potential is

$$
\begin{aligned}
V_{P} & =\frac{2 \pi \sigma}{1+6 \nu} \int_{0}^{R} \frac{r d r}{\left(z^{2}+r^{2}\right)^{\frac{1}{2}+3 \nu}} \\
& =\frac{2 \pi \sigma}{1-36 \nu^{2}}\left(\left(z^{2}+R^{2}\right)^{\frac{1}{2}-3 \nu}-z^{1-6 \nu}\right),
\end{aligned}
$$

and the electric field is

$$
E_{P}(z)=\frac{2 \pi \sigma}{1+6 \nu}\left(z^{-6 \nu}-z\left(z^{2}+R^{2}\right)^{-\frac{1}{2}-3 \nu}\right) .
$$

For a small $\nu$ we have

$$
\begin{aligned}
V & \approx 2 \pi \sigma\left(\sqrt{z^{2}+R^{2}}-z\right) \\
& +2 \pi \nu \sigma\left(6 z \ln z-3 \sqrt{z^{2}+R^{2}} \ln \left(z^{2}+R^{2}\right)\right)
\end{aligned}
$$

and

$$
\begin{aligned}
E_{P}(z) & \approx 2 \pi \sigma\left(1-\frac{z}{\sqrt{z^{2}+R^{2}}}\right)+2 \pi \nu \sigma(-6 \\
& +\frac{6 z}{\sqrt{z^{2}+R^{2}}}-6 \ln z \\
& \left.+\frac{3 z}{\sqrt{z^{2}+R^{2}}} \ln \left(R^{2}+z^{2}\right)\right) .
\end{aligned}
$$

\section{Conclusion}

According to Cavendish type experiments [15], let us consider that the radii of the two concentric spheres are $R_{1}$ and $R_{2},\left(R_{1}<R_{2}\right)$, with the charges $Q_{1}$ and $Q_{2}$ spread uniformly over them, respectively. Then, the potential at $r$ [16] is given by

$$
\begin{aligned}
V(r) & =\frac{Q_{1}}{2 R_{1} r}\left(f\left(r+R_{1}\right)-f\left(\left|r-R_{1}\right|\right)\right. \\
& +\frac{Q_{2}}{2 R_{2} r}\left(f\left(r+R_{2}\right)-f\left(\left|r-R_{2}\right|\right),\right.
\end{aligned}
$$

where $r$ is the distance from the origin ( center of concentric spheres). According to Ref. [17], we get the potential on the inner shell and outer shell as

$$
\begin{aligned}
V\left(R_{1}\right) & =\frac{Q_{1}}{2 R_{1}^{2}} f\left(2 R_{1}\right) \\
& +\frac{Q_{2}}{2 R_{1} R_{2}}\left[f\left(R_{1}+R_{2}\right)-f\left(\left|R_{2}-R_{1}\right|\right)\right] \\
V\left(R_{2}\right) & =\frac{Q_{2}}{2 R_{2}^{2}} f\left(2 R_{1}\right) \\
& +\frac{Q_{1}}{2 R_{1} R_{2}}\left[f\left(R_{1}+R_{2}\right)-f\left(\left|R_{2}-R_{1}\right|\right)\right],
\end{aligned}
$$

where

$$
f(r)=\int_{0}^{r} s U(s) d s
$$

and $U(r)$ is the electrostatic potential for a unit charge. For the ordinary electrostatics, we have $U=1 / r$, which gives $f=r$. In the deformed electrostatics with DD, from the Eq. (30), we get

$$
U=\frac{1}{1+6 \nu} \frac{1}{r^{1+6 \nu}},
$$

For small $\nu$, we have

$$
f(r) \approx r(1-6 \nu \ln r) .
$$

After the outer shell was charged by a potential $V_{0}$, a part of the charge would pass through the connecting wire into the inner shell until the equilibrium $V\left(R_{1}\right)=V\left(R_{2}\right)=V_{0}$ was reached. Then, the charges on the inner shell could be determined as

$$
V_{c}\left(R_{1}\right) \approx 6 \nu\left(\frac{R_{2}}{R_{2}-R_{1}}\right) V_{0} M\left(R_{1}, R_{2}\right),
$$

where

$$
M\left(R_{1}, R_{2}\right)=\frac{1}{2}\left[\frac{R_{2}}{R_{1}} \ln \frac{R_{2}+R_{1}}{R_{2}-R_{1}}-\ln \frac{4 R_{2}^{2}}{R_{2}^{2}-R_{1}^{2}}\right] .
$$

In the ordinary electrostatics $(\nu=0)$, we have $V_{c}\left(R_{1}\right)=0$, while $V_{c}\left(R_{1}\right) \neq 0$ for $\nu \neq 0$. So, by detecting the electrostatic potential on the inner shell directly, one could obtain the deviation from Coulomb's inverse square law, and then the ordinary derivative in the Maxwell equation could be replaced with DD.

There remains much to be studied in this direction. We should investigate the Maxwell equation with DD and derive the electromagnetic wave equation for the completion of this deformed electro-magnetics. The study of magnetic monopole with deviation seems to be possible with the help of DD. We think that these topics and their related content will be clear soon.

\section{Acknowledgement}

The authors thank the referee for a careful reading of the manuscript and constructive remarks. 
1. S. Plimpton, W. Lawton. Phys. Rev. 50 (1936) 1066. https: //doi.org/10.1103/PhysRev.50.1066

2. D. Bartlett, P. Goldhagen, E. Phillips. Phys. Rev. D 2 (1970) 483. https://doi.org/10.1103/PhysRevD.2.483

3. E. Williams, J. Faller, H. Hill. Phys. Rev. Let. 26 (1971) 721. https://doi.org/10.1103/PhysRevLett.26. 721

4. E. P. Wigner, Phys. Rev. 77 (1950) 711.https://doi.org/ 10.1103/PhysRev.77.711

5. C. Dunkl, Transactions of the American Mathematical Society 311 (1989) 167. https://doi.org/10.1090/ S0002-9947-1989-0951883-8

6. F.Calogero, J. Math. Phys. 10 (1969) 2197. https://doi. org/10.1063/1.1664821

7. F.Calogero, J. Math. Phys. 12 (1971) 419. https://doi. org/10.1063/1.1665604

8. M.A.Vasiliev, Int. J. Mod. Phys. A6 (1991) 1115. https: //doi.org/10.1142/S0217751X91000605

9. R.Chakrabarti and R.Jagannathan, J. Phys. A 27 (1994) L2777. https://doi.org/10.1088/0305-4470/ $27 / 9 / 007$
10. A.Turbiner, Phys. Lett. B. 320 (1994) 281. https://doi. org/10.1016/0370-2693(94)90657-2

11. T. Brzezinski, T. Egusquiza and A. J. Macfarlane, Phys. Lett. B311 (1993) 202. https://doi.org/10.1016/ 0370-2693(93) 90555-V

12. L. Brink, T. H.Hansson and M. A. Vasiliev, Phys. Lett. B. 286 (1992) 109. https://doi.org/10.1016/ 0370-2693(92) 90166-2

13. A. P. Polychronakos, Phys. Rev. Lett. 69 (1992) 703. https: //doi.org/10.1103/PhysRevLett.69.703

14. A. J. Macfarlane, J. Math. Phys 35 (1994) 1054. https: //doi.org/10.1063/1.530628

15. H. Cavendish, The Electrical Researches of the Honourable Henry Cavendish, ed J. Maxwell (Cambridge: Cambridge University Press, 1879).

16. Y. Zhang, Special Relativity and its Experimental Foundations (Singapore: World Scientific) (1998) pp. 248-52.

17. L. Tu and J. Luo, Metrologia 41 (2004) S136. https:// doi.org/10.1088/0026-1394/41/5/S04 